FTPI-MINN-03/30

UMN-TH-2219/03

hep-th/0311015

\title{
Stringy profiles of gauge field tadpoles near orbifold singularities: II. field theoretical implications
}

\author{
Stefan Groot Nibbelink ${ }^{a, 1}$, Mark Laidlaw ${ }^{b, 2}$, \\ a William I. Fine Theoretical Physics Institute, School of Physics 8 Astronomy, \\ University of Minnesota, 116 Church Street S.E., Minneapolis, MN 55455, USA. \\ ${ }^{b}$ Perimeter Institute for Theoretical Physics, \\ 35 King Street North, Waterloo, Ontario N2J 2W9, Canada
}

\begin{abstract}
Recent work has provided a direct string calculation of the internal coordinate dependence of gauge field tadpoles on the orbifold $\mathbb{C}^{3} / \mathbb{Z}_{3}$. We investigate the structure of these profiles in momentum and coordinate space representations using both analytical and numerical methods. The twisted states are determined to be localized within a few string lengths of the singularity. This provides an example of how delta-like singularities, which are typical for field theories on orbifolds, are avoided in string theory. A systematic expansion of the full string result allows us to speculate on how the leading stringy effects can be incorporated in a field theoretical description. In one heterotic model, we find that even though tachyons are not present in the physical spectrum, they totally dominate the tadpole profile in the vicinity of the fixed point.
\end{abstract}

\footnotetext{
$1 \quad$ E-mail: nibbelin@hep.umn.edu

2 E-mail: mlaidlaw@perimeterinstitute.ca
} 


\section{Introduction}

In this second paper in a series of two on the profiles of gauge field tadpoles in extra dimensions string and field theory, we offer a field theoretical interpretation of the string results obtained in ref. 1]. As opposed to that paper, which was written for the string oriented audience, the presentation in this paper is aimed at those interested in physics of extra dimensions and string phenomenology.

In the recent literature there has been a great deal of attention to the study of theories with extra dimensions, some inspired by the works of refs. 2, 3, 4, 5. Many of the proposed models are based on simple orbifolds like $S^{1} / \mathbb{Z}_{2}$ or $S^{1} / \mathbb{Z}_{2} \times \mathbb{Z}_{2}^{\prime}$. It is not entirely clear that field theories on orbifolds are physically well-defined: Near the orbifold singularity, strong curvature effects may lead to a breakdown of field theory computations. Moreover, theories of extra dimensions are in general nonrenormalizable, and should therefore be defined with some sort of cut-off. In this context, calculations that involve momenta much larger than this cut-off are beyond the validity of the theory. On the other hand, to probe the full structure of orbifold singularities arbitrary momenta are needed. A simple momentum cut-off also does not seem to be appropriate, since it breaks Lorentz invariance and may violate other defining symmetries of the orbifold field theory. The only consistent ultra-violet completion of field theory we have to date is string theory. It evades this problem by providing the string scale that is the natural cut-off scale for field theory [6, 7, while maintaining important symmetries like Lorentz invariance at the same time.

Fayet-Iliopoulos tadpoles on orbifolds provide a particularly interesting setting in which such consistency questions can be put forward. Four dimensional Fayet-Iliopoulos $D$-terms [8] have a natural generalization to five dimensional supersymmetric gauge theories on orbifolds. As observed in ref. 9] the effective auxiliary field at the boundaries of $S^{1} / \mathbb{Z}_{2}$ also contains a derivative with respect to the fifth dimension of a real scalar field part of the five dimensional gauge multiplet. There have been several calculations showing that such interactions are generated at the one-loop level 10, 11, 12, 13, 14. For bulk states the result is given by a massive quadratically divergent integral times delta-functions at the orbifold singularities. Here the "mass" represents the derivative squared with respect to the fifth dimension acting on these delta-functions. By investigating the required counter-term structure for this real scalar field of the gauge multiplet, it has been argued in refs. [13, 14, 15] that these tadpoles may lead to strong localization of bulk zero modes. This treatment has not been universally accepted, for alternative treatments see refs. 16, 17.

The existence of Fayet-Iliopoulos tadpoles for both auxiliary and derivative of physical fields is in no way particular to five dimensional models. One-loop computations in higher dimensional field theoretical models [18, 19] and in field theory approximation of heterotic string models [20] have confirmed that these tadpoles are generated locally at orbifold fixed points. (Fayet-Iliopoulos $D_{-}$ terms for zero modes have been discussed in heterotic [21, 22, 23, and type I string [24] context.) These results provided the main motivations to establish that such tadpoles also arise in string theory [1. The main objective of this paper is to compare the previously obtained field theory results for gauge field tadpoles with our string calculations. To this end we perform both analytical and numerical analyses of the string expressions to compare them to the field theoretical expectations. We seek to give a physical interpretation of the stringy corrections.

This paper is organized as follows: We begin in section 2 by reviewing the low energy field theory description of gauge field tadpoles in heterotic models. In section 3 we describe our results for these tadpoles obtained by direct string calculation [1] in a language chosen to make the similarities and

differences with the field theory results manifest. Section 4 is devoted to detailed studies of the 
resulting profiles of the gauge field tadpoles. We investigate which states give leading contributions: Surprisingly, we find that tachyons sometimes play an important role. Finally, we discuss some implications of our results in section 5

\section{Gauge field tadpoles in field theory}

In this section we recall some results concerning localized tadpoles in the heterotic theory obtained using field theory methods. It is based to a large extent on the results obtained in refs. 20, 25]. As we are primarily interested in local properties, we have chosen to work on the non-compact orbifold $\mathbb{C}^{3} / \mathbb{Z}_{3}$ instead of the compact orbifold $T^{6} / \mathbb{Z}_{3}$ : This avoids the introduction of another scale that obscures the tracing of the genuine stringy effects. The orbifold action $\Theta$ leads to diagonal identifications

$$
\Theta: z_{i} \rightarrow e^{2 \pi i \phi_{i}} z_{i}
$$

of the complex coordinates $z=\left(z_{i}\right)=\left(z_{1}, z_{2}, z_{3}\right)$. We consider the low-energy spectrum of the heterotic $\mathrm{E}_{8} \times \mathrm{E}_{8}{ }^{\prime}$ string on this orbifold, consisting of a ten dimensional $N=1$ supergravity multiplet and a super Yang-Mills theory with the corresponding gauge group. At the orbifold fixed point the spectrum of the theory is projected to a four dimensional $N=1$ supersymmetric theory. When we take non-trivial boundary conditions for the action of the orbifold twist on the ten dimensional gauge field one-form

$$
A(x, z) \rightarrow A(x, \Theta z)=U A(x, z) U^{-1}, \quad U=e^{2 \pi i v_{a}^{I} H_{a}^{I}},
$$

we obtain a four dimensional gauge theory with a reduced gauge group $G$ and some specific four dimensional chiral matter representations $\mathbf{R}$. Here the generators $H_{1}^{I}$ and $H_{2}^{I}$ denote the $\mathrm{SO}(16) \subset \mathrm{E}_{8}$ and $\mathrm{SO}(16)^{\prime} \subset \mathrm{E}_{8}{ }^{\prime}$ Cartan elements, respectively. The chiral matter $\mathbf{R}$ always comes in three identical

copies, because there are three internal gauge fields $A_{\underline{i}}^{\mathbf{R}}$ in the representation of the gauge group. In addition, there are four dimensional twisted states at the orbifold fixed point that also form chiral multiplets. These twisted states appear in two varieties: some come in three equal copies (their representation is denoted by $\mathbf{T}$ ), while others are singlets (referred to as $\mathbf{S}$ ). The full spectrum of the theory at the fixed point can be shown to be equivalent to one of those given in table 1.

Of the five possible models listed in table 1 only the last two have U(1)'s. The generators $q_{a}=v_{a}^{I} H_{a}^{I}$ are potentially anomalous: It can be shown that $\mathrm{U}(1)$ generated by $q_{1}$ is anomalous in the $\mathrm{E}_{7}$ model, while it is not in the $\mathrm{SU}(9)$ model. However, in that model the charge $q_{2}$ is anomalous. These U(1)'s are only anomalous at the orbifold fixed point [25], and as was investigated in 20] they can be canceled through a local version of the Green-Scharz mechanism [26]. An anomalous U(1) is expected to be spontaneously broken by a Fayet-Iliopolous tadpole for auxiliary $D$-field of the anomalous vector multiplet [21]. In ref. [20] the shape of this tadpole on the orbifold $T^{6} / \mathbb{Z}_{3}$ was calculated, and it was shown that similar tadpoles are generated for the internal gauge fields $A_{j}^{a}$ corresponding to these U(1)'s. Using the methods discussed in 27] for doing field theoretical calculations on orbifolds these results are readily extended to the non-compact orbifold $\mathbb{C}^{3} / \mathbb{Z}_{3}$ as well. In ten dimensional momentum space the result reads

$$
\left\langle A_{j}^{a}\left(k_{6}\right)\right\rangle=i k_{\underline{j}} \frac{\delta^{4}\left(k_{4}\right)}{(2 \pi)^{4}} \int \frac{d^{4} p_{4}}{(2 \pi)^{4}}\left\{\frac{3 \operatorname{tr}_{\mathbf{R}}\left(q_{a}\right)}{27} \frac{1}{p_{4}^{2}+\frac{1}{3} k_{i} k_{\underline{i}}}+\left(3 \operatorname{tr}_{\mathbf{T}}\left(q_{a}\right)+\operatorname{tr}_{\mathbf{S}}\left(q_{a}\right)\right) \frac{1}{p_{4}^{2}}\right\} .
$$




\begin{tabular}{|c|c|c|c|c|}
\hline Model & $\begin{array}{l}\text { Shift }\left(v_{1}^{I} \mid v_{2}^{I}\right) \text { and } \\
\text { gauge group } G\end{array}$ & $\begin{array}{l}\text { Untwisted } \\
\left(\mathbf{3}_{H}, \mathbf{R}\right)\end{array}$ & $\begin{array}{l}\text { Twisted } \\
\left(\mathbf{1}_{H}, \mathbf{S}\right)\end{array}$ & $\left(\overline{\mathbf{3}}_{H}, \mathbf{T}\right)$ \\
\hline $\mathrm{E}_{8}$ & $\begin{array}{cc}\frac{1}{3}\left(0^{8}\right. & \mid 0^{8} \\
& \mathrm{E}_{8} \times \mathrm{E}_{8}^{\prime}\end{array}$ & & & $(\mathbf{1})(\mathbf{1})^{\prime}$ \\
\hline $\mathrm{E}_{6}$ & $\begin{array}{c}\frac{1}{3}\left(-2,1^{2}, 0^{5} \mid 0^{8}\right) \\
\mathrm{E}_{6} \times \mathrm{SU}(3) \times \mathrm{E}_{8}^{\prime}\end{array}$ & $(\mathbf{2 7}, \overline{\mathbf{3}})(\mathbf{1})^{\prime}$ & $(27, \mathbf{1})(\mathbf{1})^{\prime}$ & $(\mathbf{1}, \mathbf{3})(1)^{\prime}$ \\
\hline $\mathrm{E}_{6}{ }^{2}$ & $\begin{array}{c}\frac{1}{3}\left(-2,1^{2}, 0^{5} \mid-2,1^{2}, 0^{5}\right) \\
\mathrm{E}_{6} \times \mathrm{SU}(3) \times \mathrm{E}_{6}{ }^{\prime} \times \mathrm{SU}(3)^{\prime}\end{array}$ & $(\mathbf{2 7}, \overline{\mathbf{3}})(\mathbf{1}, \mathbf{1})+(\mathbf{1}, \mathbf{1})(\mathbf{2 7}, \overline{\mathbf{3}})^{\prime}$ & $(1,3)(1,3)^{\prime}$ & \\
\hline $\mathrm{E}_{7}$ & $\begin{aligned} \frac{1}{3}\left(0,1^{2}, 0^{5}\right. & \left.\mid-2,0^{7}\right) \\
\mathrm{E}_{7} \times \mathrm{U}(1) & \times \mathrm{SO}(14)^{\prime} \times \mathrm{U}(1)^{\prime}\end{aligned}$ & $\begin{array}{l}(\mathbf{1})_{0}(\mathbf{6 4})_{\frac{1}{2}}^{\prime}+(\mathbf{5 6})_{1}(\mathbf{1})_{0}^{\prime} \\
+(\mathbf{1})_{0}(\mathbf{1 4})_{-1}^{\prime}+(\mathbf{1})_{-2}(\mathbf{1})_{0}^{\prime}\end{array}$ & $\begin{array}{l}(1)_{\frac{2}{3}}(14)_{-\frac{1}{3}}^{\prime} \\
+(\mathbf{1})_{-\frac{4}{3}}(\mathbf{1})_{\frac{2}{3}}^{\prime}\end{array}$ & $(\mathbf{1})_{\frac{2}{3}}(\mathbf{1})_{\frac{2}{3}}^{\prime}$ \\
\hline $\mathrm{SU}(9)$ & 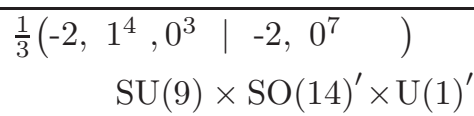 & $\begin{array}{l}(\mathbf{8 4})(\mathbf{1})_{0}^{\prime}+(\mathbf{1})(\mathbf{6 4})_{\frac{1}{2}}^{\prime} \\
+(\mathbf{1})(\mathbf{1 4})_{-1}^{\prime}\end{array}$ & $(\overline{\mathbf{9}})(\mathbf{1})_{\frac{2}{3}}^{\prime}$ & \\
\hline
\end{tabular}

Table 1: The spectra at the fixed point of the five $\mathbb{Z}_{3}$ orbifold models are displayed.

The trace of charge $q_{a}$ over representation $\mathbf{r}$ is denoted by $\operatorname{tr}_{\mathbf{r}}\left(q_{a}\right)$. The four dimensional delta $\delta^{4}\left(k_{4}\right)$ is a consequence of four dimensional momentum conservation. All complex internal momenta $k_{6}=\left(k_{i}, k_{\underline{i}}\right)$ are allowed, because this expression is the Fourier transform of (derivatives of) the delta function $\delta^{6}(z)$ at the orbifold fixed point. The four dimensional momentum integrals are clearly divergent, and in particular the leading (quadratic) divergence is proportional to the trace of the charges over the full spectrum at the fixed point. This quadratic divergence is present only when there is an anomalous $U(1)$ in the spectrum [21].

The four dimensional divergences can be regularized in various ways. We follow [6] and use a variant of Schwinger's proper time regularization in order to make contact with the string calculation of the next section. We write

$$
\int \frac{\mathrm{d}^{4} p_{4}}{p_{4}^{2}+m^{2}}=\frac{\pi}{4} \Lambda^{2} \int_{-\frac{1}{2}}^{\frac{1}{2}} \mathrm{~d} \tau_{1} \int_{1}^{\infty} \frac{\mathrm{d} \tau_{2}}{\tau_{2}^{2}} e^{-4 \pi \tau_{2} m^{2} / \Lambda^{2}},
$$

for an arbitrary mass $m$ and a cut-off scale $\Lambda$. The $\tau_{1}$ integration is clearly redundant, but it has been included in this formula for the subsequent comparison with the string theory tadpole. Using the proper time representation of the momentum integrals the expression for the tadpole may be written as:

$$
\left\langle F_{j \underline{j}}^{b}(k)\right\rangle=\frac{\delta^{4}\left(k_{4}\right)}{(2 \pi)^{4}} \frac{\pi}{4} \Lambda^{2} \int_{-\frac{1}{2}}^{\frac{1}{2}} \mathrm{~d} \tau_{1} \int_{1}^{\infty} \frac{\mathrm{d} \tau_{2}}{\tau_{2}^{2}} \sum_{s=u n, t w} Q_{s}^{b} e^{-\Delta_{s} k_{i} k_{\underline{i}} / \Lambda^{2}} .
$$

Here we have combined tadpoles for $A_{j}^{b}$ and $A_{j}^{b}$ after a partial integration to obtain the field strength $F_{j \underline{j}}^{b}$. The quantities $Q_{u n}^{b}, Q_{t w}^{b}, \Delta_{u n}$ and $\Delta_{t w}$ appearing in this expression are given by

$$
Q_{u n}^{b}=\frac{3}{27} \operatorname{tr}_{\mathbf{R}}\left(q_{b}\right), \quad Q_{t w}^{b}=\operatorname{tr}_{\mathbf{S}}\left(q_{b}\right)+3 \operatorname{tr}_{\mathbf{T}}\left(q_{b}\right), \quad \Delta_{u n}=4 \pi \tau_{2} \frac{1}{3}, \quad \Delta_{t w}=0 .
$$


The subscripts $u n$ and $t w$ refer respectively to the untwisted and twisted sectors. The width $\Delta_{s}$ could in principle carry internal space indices $i$ or $\underline{i}$, but rotational symmetry of the orbifold requires them all to be the same. For the untwisted sector $\Delta_{u n} \neq 0$ and the tadpole takes the form of Gaussian distributions with widths depending on $\tau_{2}$. This variable is integrated up to the cut-off of the effective field theory, which has be scaled to unity by pulling out $\Lambda$ explicitly in equation 4 . In particular for large internal momenta $k_{6}$ the tadpole will be damped considerably. For the fixed point states there is no such suppression because $\Delta_{t w}=0$. This reflects the fact that in field theory we have treated the twisted states as fields that are localized exactly at the fixed points. In the next section we will see that the string theory treatment of these states is fundamentally different.

In order to have an exact measure of when and to what extent states are localized we introduce the concept of localization width, defined as

$$
\overline{\Delta_{s}}=\int_{-\frac{1}{2}}^{\frac{1}{2}} \mathrm{~d} \tau_{1} \int_{1}^{\infty} \frac{\mathrm{d} \tau_{2}}{\tau_{2}^{2}} \Delta_{s}\left(\tau_{1}, \tau_{2}\right) .
$$

One can think of this quantity as the average radial spread of a given state around the orbifold fixed point. A bulk, or delocalized, state has a (logarithmically) divergent localization width, while this width is zero for a fixed point state. It is not difficult to see that this definition precisely matches with the interpretation of the untwisted and twisted states in the field theory treatment, since we obviously have $\overline{\Delta_{u n}}=\infty$ and $\overline{\Delta_{t w}}=0$, respectively.

\section{Gauge field tadpoles in string theory}

The gauge field tadpoles, which were described from a field theory point of view, also arise at one loop in the full heterotic string theory. In this paper we wish to compare the field and string theory results to each other, therefore we discuss the schematic structure of the string theory tadpoles here. Details of the string computation are given in ref. [1. Here we only call to the attention of the reader those ingredients that are essential to the understanding of the analysis of the tadpoles that we present below.

A one loop calculation of a tadpole in closed string theory involves averaging the corresponding vertex operator over inequivalent torus world sheets. A two dimensional torus is defined as the complex plane $\mathbb{C}$ divided by the lattice spanned by 1 and the complex number $\tau=\tau_{1}+i \tau_{2}$. Only by restricting this (modular) parameter $\tau$ to a fundamental domain, the inequivalent tori are labeled uniquely. In figure 1 we have depicted the fundamental domain $\mathcal{F}$ which is defined by $|\tau| \geq 1$ and $-\frac{1}{2} \leq \tau_{1} \leq \frac{1}{2}$. This corresponds to the most common choice for the fundamental domain in string theory. The field theory cut-off at the string scale would be given by $-\frac{1}{2} \leq \tau_{1} \leq \frac{1}{2}$ and $\tau_{2} \geq 1$, as we have discussed in the previous section.

The $\mathbb{Z}_{3}$ orbifolding leads to a classification of string states in several sectors which are determined by the periodicities of the coordinate fields under the lattice shifts 1 and $\tau$. These can be characterized broadly as untwisted and twisted sectors. The untwisted states (the $u$-sector) have trivial boundary conditions under the shift 1 . We introduce a finer classification of the twisted states that distinguishes between those with trivial boundary conditions under the shift $\tau$ (the $t$-sector) and those with nontrivial boundary conditions in both directions (the $d_{ \pm}$-sectors). The distinction between these doubly twisted states is whether they have the same or opposite orbifold conditions under both lattice shifts. 


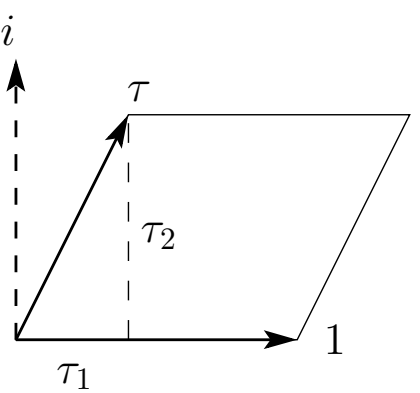

world sheet torus

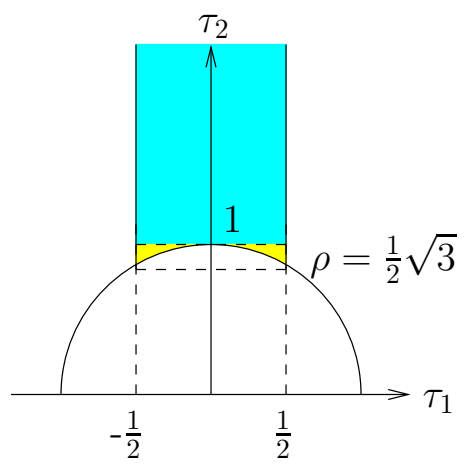

$\mathcal{F}=\left\{\tau \in \mathbb{C} \mid-\frac{1}{2}<\tau_{1}<\frac{1}{2}, \tau_{2}>\sqrt{1-\tau_{1}^{2}}\right\}$,

Figure 1: The first picture gives a torus defined by the complex variable $\tau$. To only label inequivalent tori this parameter is restricted to the fundamental domain $\mathcal{F}$, depicted in the second picture.

In our classification the $u$-sector corresponds to the untwisted field theory states (un), and the $t$ - and $d_{ \pm}$-sectors are the twisted states $(t w)$. The gauge field tadpole takes the form of a sum over these different sectors

$$
\left\langle F_{j \underline{j}}^{b}\left(k_{6}\right)\right\rangle=\frac{\delta^{4}\left(k_{4}\right)}{(2 \pi)^{4}} \sum_{s=u, t, d_{ \pm}} G_{s}^{b}\left(k_{6}\right), \quad G_{s}^{b}\left(k_{6}\right)=\int_{\mathcal{F}} \frac{\mathrm{d}^{2} \tau}{\tau_{2}^{2}} Q_{s}^{b}(\tau) e^{-\Delta_{s}(\tau, \bar{\tau}) k_{i} k_{\underline{i}}} .
$$

In this expression $Q_{s}^{b}(\tau)$ can be thought of as the trace of the $q_{b}$ charges of sector $s$. In string theory the orbifold space coordinates $X^{i}$ and $X^{i}$ are fields on the torus world sheet. Their expectation value $\Delta_{s}(\tau, \bar{\tau})=\left\langle X^{\underline{i}} X^{i}\right\rangle_{s}(\tau, \bar{\tau})$ set the widths of the orbifold singularity for the states of sector $s$. This interpretation is motivated by the observation that these expectation values appear as the (inverse) standard deviations of the Gaussian distributions for the internal momentum $k$ of the gauge field. (Here we have dropped the subscript on the internal momenta $k_{6}$.) Rotational symmetry requires that the widths $\Delta_{s}(\tau, \bar{\tau})$ are equal in all three complex momentum directions for each sector. The exact one loop string expression for the functions $Q_{s}^{b}(\tau)$ and $\Delta_{s}(\tau, \bar{\tau})$ have been obtained in ref. [1]. The notation of this expression (8) emphasizes that $Q_{s}^{b}(\tau)$ is holomorphic in $\tau$ and that $\Delta_{s}(\tau, \bar{\tau})$ is a real function of both $\tau$ and $\bar{\tau}$. For this reason it is necessary to take care when interpreting these quantities (in field theory). We emphasise that even though $Q_{s}^{b}(\tau)$ is a complex function, the tadpole (8) is real, because the integration over the fundamental domain is symmetric under $\tau_{1} \rightarrow-\tau_{1}$.

The field theoretical expression (15) for the gauge field tadpole has a structure compatible with that of string theory (8). But even at this crude level some important differences can be identified: In field theory we only distinguish between untwisted (un) and twisted states $(t w)$; the string classification is finer since the twisted states are now divided up into single $(t)$ and doubly twisted $\left(d_{ \pm}\right)$states, which have different profiles over the extra dimensions as we will demonstrate in detail in section 4 In addition, the integration over $\tau_{1}$ and $\tau_{2}$ is different: In string theory the lower bound of $\tau_{2}$ depends on $\tau_{1}$, because of the shape of the fundamental domain given in figure 1 while in field theory it is equal to unity. Neglecting the peculiar shape of the fundamental domain gives an error of the order of $\pi / 3-1 \approx 0.047$ (taking the factor $1 / \tau_{2}^{2}$ as part of the integration measure). There is no explicit cut-off dependence in the string result because we have chosen string units: $\Lambda=M_{s}=1$. 


\begin{tabular}{|c|cccl|}
\hline$\tau_{2}$ & $\frac{1}{2} \sqrt{3}$ & 1 & \multicolumn{1}{c}{1.5} & \multicolumn{1}{c|}{2} \\
\hline$e^{-2 \pi \frac{1}{3} \tau_{2}}$ & 0.163 & 0.123 & 0.043 & 0.015 \\
$e^{-2 \pi \frac{2}{3} \tau_{2}}$ & 0.027 & 0.015 & 0.002 & 0.0002 \\
$e^{-2 \pi \tau_{2}}$ & 0.004 & 0.002 & 0.0001 & 0.00003 \\
\hline
\end{tabular}

Table 2: In this table we have tabulated the values of first few exponential factors in $\tau_{2}$ that arise in the expansions of $Q_{s}(\tau)$ and $\Delta_{s}(\tau, \bar{\tau})$ for a $\mathbb{Z}_{3}$ orbifold. As these factors are quite small, it is to be expected that the first few terms give a reasonably accurate approximation of the full string results.

The tadpole takes the form in momentum space of a sum of Gaussian distributions with $\tau$ dependent standard deviations $\Delta_{s}$, it is straightforward to obtain the Fourier transform to the internal coordinate space

$$
\left\langle F_{j \underline{j}}^{b}(z)\right\rangle=\frac{\delta^{4}\left(k_{4}\right)}{(2 \pi)^{4}} \sum_{s=u, t, d_{ \pm}} \mathcal{G}_{s}^{b}(z), \quad \mathcal{G}_{s}^{b}(z)=\int_{\mathcal{F}} \frac{\mathrm{d}^{2} \tau}{\tau_{2}^{2}} Q_{s}^{b}(\tau)\left(\frac{1}{\pi \Delta_{s}(\tau, \bar{\tau})}\right)^{3} e^{-\bar{z} z / \Delta_{s}(\tau, \bar{\tau})} .
$$

It should, however, be stressed that the Fourier transform only exists when all $\Delta_{s}(\tau) \geq 0$ for all $\tau$.

To gain more insight into the relationship between the field and string predictions for the gauge field tadpoles, we systematically approximate the string results to an accuracy that is roughly of the same order as the field theory approximation of the fundamental domain of the string. The functions $Q_{s}(\tau)$ and $\Delta_{s}(\tau, \bar{\tau})$ can both be written as a power series in $\exp \left(-2 \pi \tau_{2}\right)$. Due to the $\mathbb{Z}_{3}$ orbifolding fractional powers $1 / 3$ and $2 / 3$ of this exponential factor also arise. Since $\tau$ is restricted to take values within the fundamental domain, $\tau_{2} \geq \rho=\frac{1}{2} \sqrt{3}$, and consequently the exponential factor $\exp \left(-2 \pi \tau_{2}\right)$ always gives a sizable suppression even when $\tau_{2}$ is not very large. We have evaluated those exponentials for various small values of $\tau_{2}$ in table 2. The table shows that corrections of the order of $\exp \left(-2 \pi \tau_{2}\right)$ are at least suppressed by a factor 0.004 , which is an order of magnitude smaller than the factor of 0.05 associated with the field theory motivated truncation of the fundamental domain.

Motivated by this, we expand the exact expressions for $\Delta_{s}(\tau, \bar{\tau})$ obtained in ref. [1] to order $\exp \left(-2 \pi \tau_{2}\right)$ :

$$
\begin{aligned}
& \Delta_{u}(\tau, \bar{\tau})=c-3 \ln 3+4 \pi \tau_{2} \frac{1}{3}+\ldots, \\
& \Delta_{t}(\tau, \bar{\tau})=c+6 \cos \left(2 \pi \frac{\tau_{1}}{3}\right) e^{-2 \pi \tau_{2} \frac{1}{3}}+9 \cos \left(4 \pi \frac{\tau_{1}}{3}\right) e^{-2 \pi \tau_{2} \frac{2}{3}}+\ldots, \\
& \Delta_{d_{+}}(\tau, \bar{\tau})=c+6 \cos \left(2 \pi \frac{\tau_{1}-1}{3}\right) e^{-2 \pi \tau_{2} \frac{1}{3}}+9 \cos \left(4 \pi \frac{\tau_{1}-1}{3}\right) e^{-2 \pi \tau_{2} \frac{2}{3}}+\ldots, \\
& \Delta_{d_{-}}(\tau, \bar{\tau})=c+6 \cos \left(2 \pi \frac{\tau_{1}+1}{3}\right) e^{-2 \pi \tau_{2}} \frac{1}{3}+9 \cos \left(4 \pi \frac{\tau_{1}+1}{3}\right) e^{-2 \pi \tau_{2} \frac{2}{3}}+\ldots
\end{aligned}
$$

The constant $c$ is related to the normal ordering constant $\tilde{c}$ that appeared in string calculation of the tadpole in ref. [1]; the redefinition $c=\tilde{c}-\ln 3$ turns out to be convenient since then all $\Delta_{s}$ with $s=t, d_{ \pm}$in (10) contain just a single constant term. (All functions $\Delta_{s}$ are expressed as a sum of untwisted propagators on the string world sheet at zero separation. A logarithmically divergent terms has to be subtracted in this limit, which introduces the arbitrary constant $\tilde{c}$, as we have discussed in 11.) It should also be stressed that the approximation for $\Delta_{u}(\tau, \bar{\tau})$ is exact up to order $\exp \left(-2 \pi \tau_{2}\right)$; 
in particular it does not contain any order $\exp \left(-2 \pi \tau_{2} / 3\right)$ or $\exp \left(-4 \pi \tau_{2} / 3\right)$ terms. This shows that the field theory approximation of $\Delta_{u}$ given in (6) is very accurate, with the first corrections appearing at order $e^{-2 \pi \tau_{2}}$. Similarly the reason why field theory fails to notice the exponentially decaying corrections to the twisted propagators can easily be understood: The field theory is expected to be accurate only for the infra-red region $\tau_{2} \gg 1$. Apart from the exponentially suppressed corrections to the (twisted) propagators, we see that the propagators depend on $\tau_{1}$. This dependence makes a distinction between the various boundary conditions in the twisted sectors.

Apart from these stringy corrections that decay exponentially with $\tau_{2}$, two constants have appear in the expansion (10): The string normal ordering constant $c$ and $-3 \ln 3$. The latter is a consequence of the $\mathbb{Z}_{3}$ orbifold which we are considering throughout this work. As far as we know there are no fundamental principles in string theory that could fix the value of the normal ordering constant. However, as we observed in [1], the Fourier transform of the Gaussians in (8) to configuration space (92) is only well-defined provided that all functions $\Delta_{s}$ never turn negative. This leads to a lower bound $c_{0}$ for the normal ordering constant $c$. From the formulae (10) we find the approximate bound

$$
c \geq c_{0}=6 e^{-2 \pi \frac{1}{3} \rho}-9 e^{-2 \pi \frac{2}{3} \rho}+\ldots \approx 0.75824 .
$$

(The numerical value is obtained using the exact string expressions evaluated at $\rho=\frac{1}{2} \sqrt{3}$.) Moreover, from the approximations we infer that all twisted correlators approach the normal ordering constant $c$ for large $\tau_{2}$. This leads to one of the most fundamental differences between string and field theory: In field theory the twisted states have constant profiles in $k$, while in string theory they always decay exponentially (as $\exp \left(|k|^{2}\right)$ ) precisely because this constant is strictly positive: $c \geq c_{0} \approx 0.75824>0$. In addition, the function $\Delta_{u}$ includes the constant part $c-3 \ln 3$ which is absent in field theory, as is apparent from comparing (10) with (6).

We have plotted the functions $\Delta_{s}\left(\tau_{1}, \tau_{2}\right)$ for the different sectors $s=u, t, d_{+}$and $d_{-}$in figure2 for five values $\tau_{1}$. It is important to note that the behavior of the untwisted states $(u)$ is very different than that of the twisted states $\left(t\right.$, and $\left.d_{ \pm}\right)$for large $\tau_{2}$ : The former grow linearly with $\tau_{2}$, while the latter all approach the constant $c_{0}$, as expected from approximations (10). The function $\Delta_{t}$ is symmetric under the sign flip of $\tau_{1} \rightarrow-\tau_{1}$, and that

$$
\Delta_{d_{+}}\left(-\tau_{1}, \tau_{2}\right)=\Delta_{d_{-}}\left(\tau_{1}, \tau_{2}\right)
$$

(Using the exact expressions for these functions derived in [1], these properties can be verified analytically.) In the plots 2 we have chosen the string normal ordering constant equal to the minimum value for which all propagators for all $\tau$ are positive definite. If we take $c$ larger than $c_{0}$, all curves are shifted upwards by the same amount.

In direct analogy to our field theory definition (7), we introduce localization widths for the different sectors as

$$
\overline{\Delta_{s}}=\frac{3}{2 \pi} \int_{\mathcal{F}} \frac{\mathrm{d}^{2} \tau}{\tau_{2}^{2}} \Delta_{s}\left(\tau_{1}, \tau_{2}\right),
$$

and the normalization factor $3 /(2 \pi)$ has been included to ensure the interpretation of an average. Notice that as in the field theory case $\overline{\Delta_{u}}$ is logarithmically divergent. This is another indication that string theory treats the untwisted states as genuine bulk states. Furthermore, it is obvious that larger values of the normal ordering constant $c$ correspond to larger localization widths for all fields: Larger 

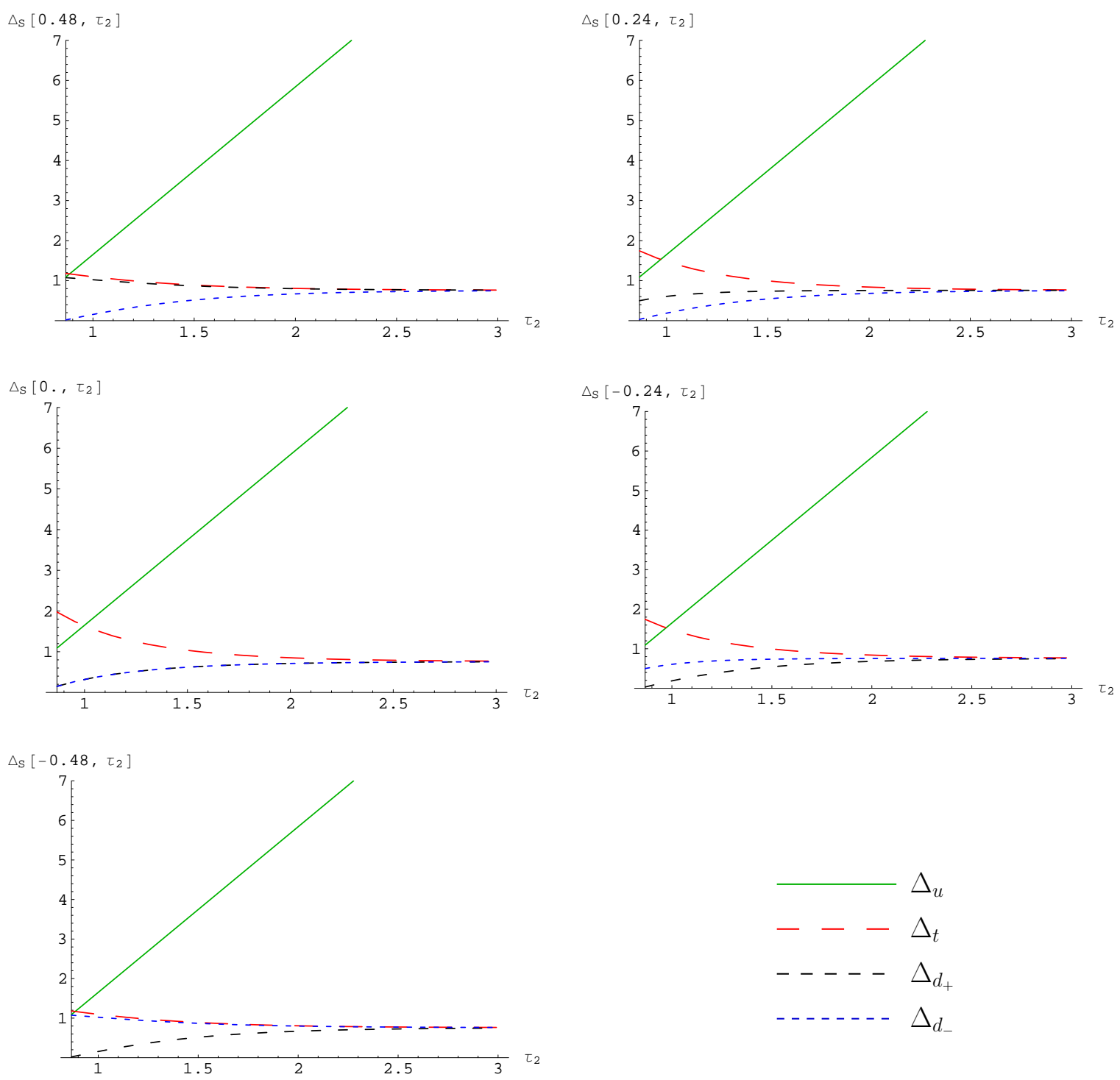

Figure 2: The five plots show the differences of the functions $\Delta_{s}\left(\tau_{1}, \tau_{2}\right)$ for the values $\tau_{1}=$ $-0.48,-0.24,0,0.24$ and 0.48 in the different sectors $s=u, t, d_{+}$and $d_{-}$. (The values were mainly motivated to show the changes in $\Delta_{s}$ with $\tau_{1}$ clearly, and to avoid too many coincident curves.) The change of the function $\Delta_{u}$ for these values of $\tau_{1}$ is hardly visible as can be seen from its approximation in equation (10). The roles of $\Delta_{d_{+}}$and $\Delta_{d_{-}}$are interchanged when we take $\tau_{1} \rightarrow-\tau_{1}$. 

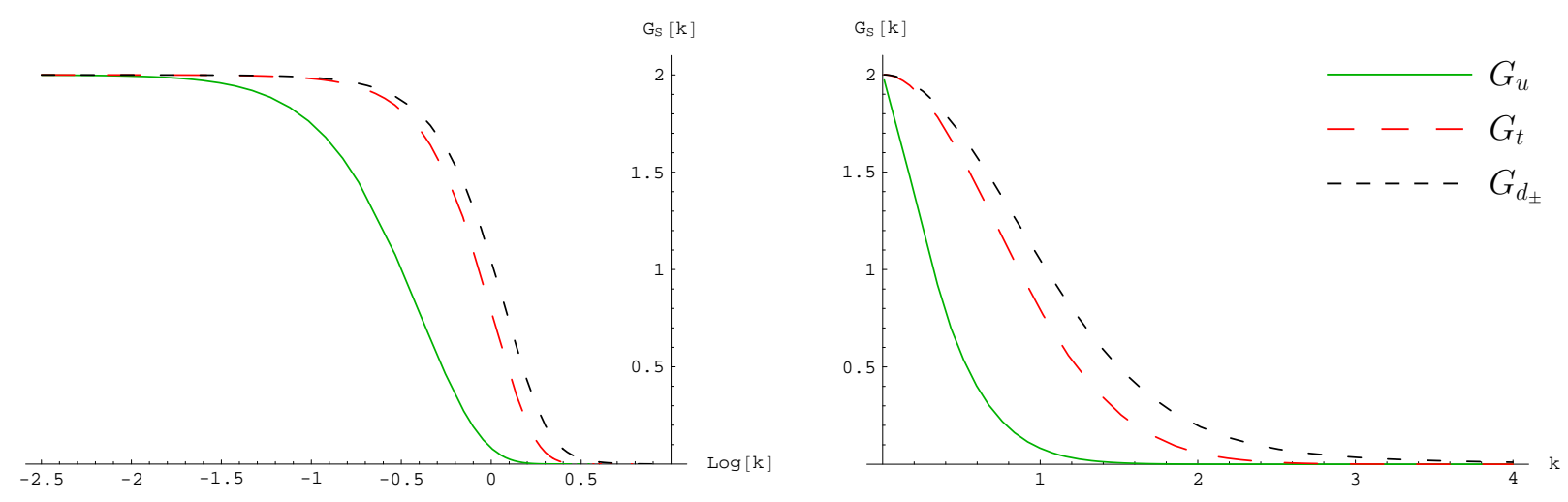

Figure 3: These two figures display the momentum profiles for the $u, t$ and $d_{ \pm}$-sector. The picture on the left is plotted using a (decimal) log-scale; the picture on the right uses a linear scale.

$c$ corresponds to more delocalized effects of the fixed point fields. We observe that choosing $c$ to take its minimum value $c_{0}$ gives localizations that are the closest to the naive field theory expectation. In that case we find

$$
\overline{\Delta_{u}}=\infty, \quad \overline{\Delta_{t}} \approx 1.98627, \quad \overline{\Delta_{d_{+}}}=\overline{\Delta_{d_{-}}} \approx 1.38752 .
$$

Since the twisted localization widths are quite close to unity, the spread of these states is of the order of the string scale. The hierarchy between $\overline{\Delta_{u}}, \overline{\Delta_{t}}$ and $\overline{\Delta_{d_{ \pm}}}$can be easily understood qualitatively: From either the approximate formulae (10) or from the plots in figure 2 we see that for most values of $\tau$ we have $\Delta_{u}(\tau)>\Delta_{t}(\rho)>\Delta_{d_{ \pm}}(\tau)$, hence similar inequalities hold for their averages.

\section{Shape of tadpoles}

In this section we investigate the different profiles for gauge field tadpoles that arise at the one-loop level in string theory. In particular we wish to examine how the twisted states contribute in the neighborhood of the orbifold singularity to give us some insight as to how fixed point states should be treated in field theory.

The general form (8) shows that there are two functions that determine the profile of the tadpole, the first is the regularized two point function of the coordinate fields $\Delta_{s}$, and the second is the charge trace $Q_{s}^{b}$ defined in (9). In contrast to the universal nature of these widths $\Delta_{s}$, which only depend on the orbifold under investigation, the charges $Q_{s}^{b}$ are very model dependent. For the $\mathbb{Z}_{3}$ orbifolds we consider there are only two different models that have anomalous $\mathrm{U}(1)$ and non-vanishing charge traces [1. We consider the $\mathrm{SU}(9)$ model first. It is the simpler of the two cases, and it will illustrate the generic properties of the tadpole profiles. With the $\mathrm{SU}(9)$ model understood we can contrast it with the $\mathrm{E}_{7}$ model which exhibits some surprising features. 


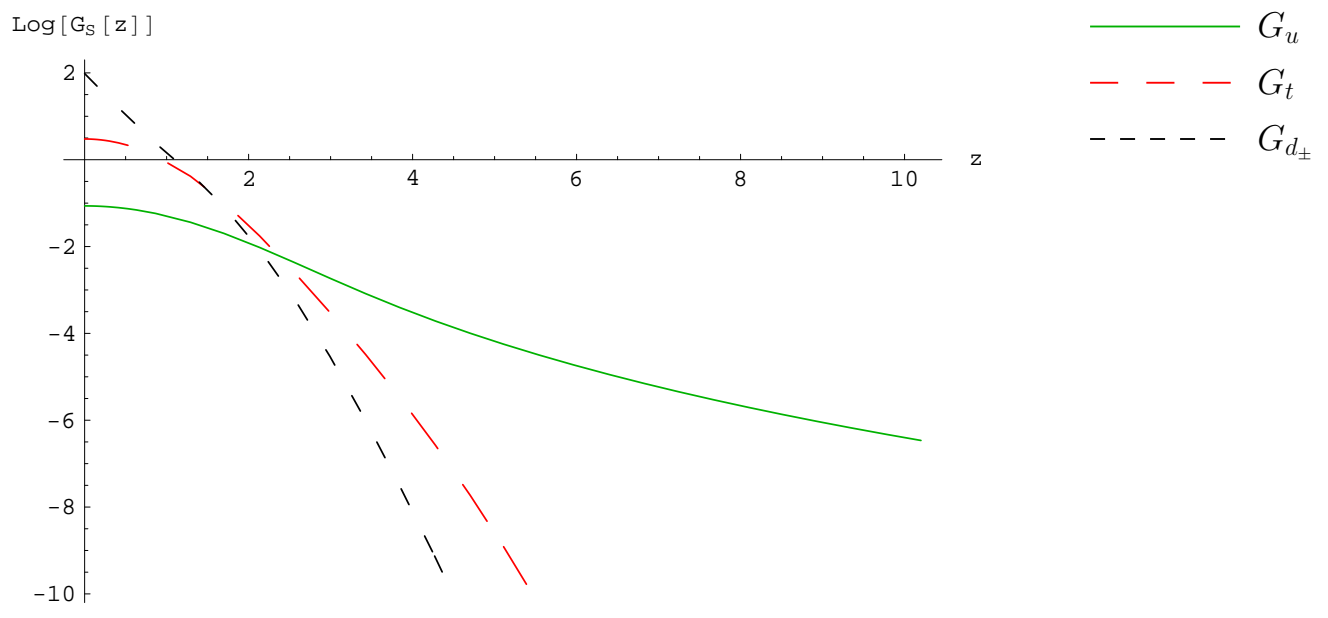

Figure 4: The spatial extension of the tadpole contributions in the sectors $u, t$ and $d_{ \pm}$are displayed on a logarithmic scale. The curve for the $u$-sector fall off much slower than the others for large values of $|z|$, as only it corresponds to bulk contributions.

\section{SU(9) model}

For the $\mathbb{Z}_{3}$ model containing the gauge group $\mathrm{SU}(9)$ the charge functions $Q_{s}^{b}$ are exactly constant and all equal within each $\mathrm{E}_{8}$ :

$$
Q_{u}^{1}=Q_{t}^{1}=Q_{d_{+}}^{1}=Q_{d_{-}}^{1}=0, \quad Q_{u}^{2}=Q_{t}^{2}=Q_{d_{+}}^{2}=Q_{d_{-}}^{2}=2 .
$$

We interpret this result as implying that all contributions, excluding the zero modes, exactly cancel each other in the loop in each sector. This shows that for this model an effective field theory approach to string theory, keeping only the string zero modes, takes all contributing states into account. We focus only on the non-vanishing charge functions in the second $\mathrm{E}_{8}$. A nice (accidental) feature of this model, which we will exploit for illustrative purposes, is that the charges $Q_{s}^{2}$ are all equal. This allows us to isolate the differences between the various sectors that arise due to the differences in the functions $\Delta_{s}$ only. In figure 3 the momentum profiles for the untwisted $(u)$ states and twisted $\left(t, d_{ \pm}\right)$ states are plotted. We see that the untwisted states are more suppressed for higher momentum than the twisted states. The field theory approximation of the contribution from twisted states is equal for any value of the internal momenta $k$, while the string results always damps momenta much larger than the string scale.

In the coordinate space representation we find more pronounced differences between the drop off in the sectors. In figure 4 we have plotted the profiles as a function of the radial variable $|z|$ in the six internal dimensions. Close to the orbifold singularity the twisted states in the $t$ and $d_{ \pm}$-sectors dominate the tadpole. At a distance of about $z=2.5$ string length all three sectors contribute with comparable magnitude. After that the curves corresponding to the $t$ and $d_{ \pm}$-sectors fall of much faster than the one for the $u$-sector. These differences are consistent with our understanding that the $u$-sector corresponds to bulk states, while the $t$ and $d_{ \pm}$-sectors constitute the fixed point states.

The difference between the plots can be understood because equation (9) contains factors $1 / \Delta_{s}$ both in the overall normalization and in the exponential. This implies that the smallest values of $\Delta_{s}$ 


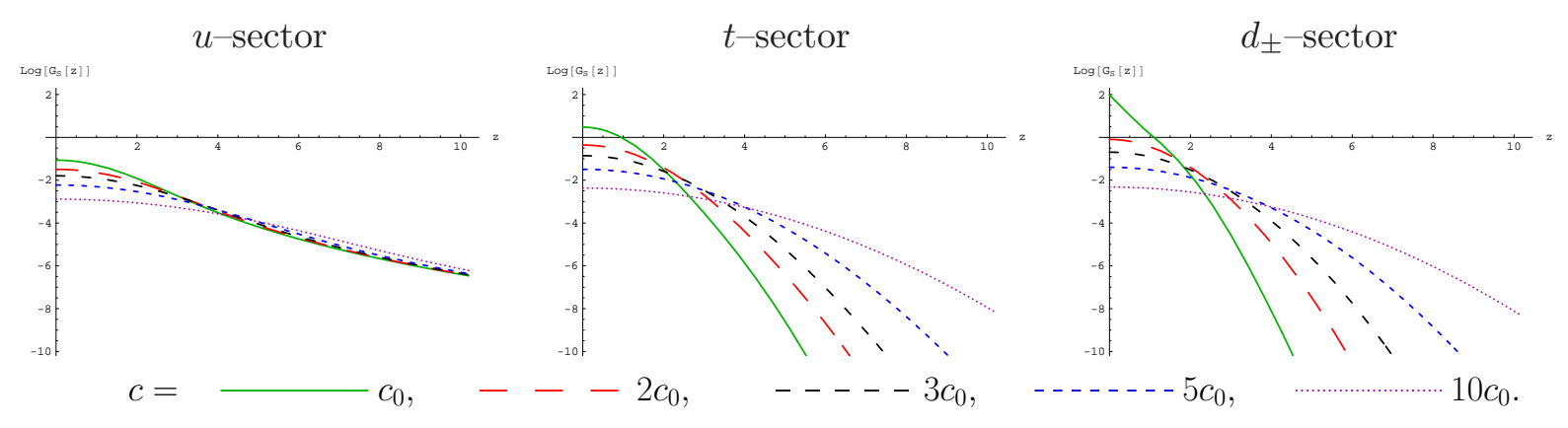

Figure 5: In this plot we compare the shapes tadpoles that arise when we take the normal ordering constant $c$ equal to $c_{0}, 2 c_{0}, 3 c_{0}, 5 c_{0}$ or $10 c_{0}$, with $c_{0}$ the minimum value of the normal ordering constant (111). We see that the larger the value of $c$ the more the profiles of the different sectors are suppressed for small values of $|z|$, while at the same time the twisted state contributions become more delocalized.

will contribute the most to the tadpoles in the coordinate space representation. However, a smaller value of the function $\Delta_{s}$ will give a much stronger suppression from the exponential for sizable values of $|z|$. Since $\Delta_{u}$ is the largest of the functions $\Delta_{s}$, as can be verified by inspecting the plots 2 the profile $\mathcal{G}_{u}(z)$ is least suppressed for large values of $|z|$. The situation close to the orbifold singularity $(|z| \approx 0)$, is the opposite, because then the exponential factor approaches unity. The values of $\mathcal{G}_{s}$ there are determined by the normalizations $1 / \Delta_{s}^{3}$, hence close to the singularity the sector $d_{ \pm}$dominates.

We can also investigate how the profiles of the tadpoles in the coordinate representation are affected by the value of the normal ordering constant. We have plotted these profiles for a range of $c$ in figure 5. The overall effects are that close to the orbifold singularity, all the profiles drop significantly for large values of $c$. When $|z|$ becomes of the order of two to four string lengths, the curves are falling off less fast for larger values of the normal ordering constant. For very large $|z|$ the contribution of the untwisted sector become identical irrespective of what the normal ordering constant is.

These features can be understood by noting that all the curves in figure 5 correspond to normalized Gaussians integrated over the fundamental domain: When integrated over $\mathbb{C}^{3} / \mathbb{Z}_{3}$ all curves give the same value (which is equal to 2 in the $\mathrm{SU}(9)$ model). From their definitions (9) it follows that for larger values of the normal ordering constant, the tadpole are smaller because the factor $1 / \Delta_{s}^{3}$ gives a bigger suppression near $z=0$, where the exponent can be neglected. But since these profiles are normalized, they need to become larger when $|z|$ is increased. Hence, as we would have expected from the localization widths (14), the profiles of the twisted states drop off slower for the larger value of c. A similar argument applies to the untwisted states: Since their localization width is infinite, the integral over the orbifold receives significant contributions for any value of $|z|$. Therefore to respect the normalization condition all untwisted curves need to tend to each other for large $|z|$. This analysis provides a different argument that taking $c=c_{0}$ leads to physics that most closely resembles the field theory treatment of the twisted states, as they are the most localized to the orbifold fixed points.

\section{$\mathrm{E}_{7}$ model}

Next, we compare the situation of the $\mathrm{SU}(9)$ model with the other anomalous model: The $\mathrm{E}_{7}$ has two $\mathrm{U}(1)$ factors in its zero mode gauge group, see table 1 The expressions for $Q_{s}^{b}$ for the $\mathrm{E}_{7}$ 
model are more complicated than those of the $\mathrm{SU}(9)$ model and we give their expansions up to order $q=\exp (2 \pi i \tau)=\exp \left(2 \pi i \tau_{1}\right) \exp \left(-2 \pi \tau_{2}\right)$

$$
\begin{aligned}
& Q_{u}^{1} \approx 3+\ldots, \\
& Q_{t}^{1} \approx \frac{1}{9} q^{-\frac{1}{3}}+\frac{5}{3}+6 q^{\frac{1}{3}}-\frac{76}{9} q^{\frac{2}{3}}+\ldots \\
& Q_{d_{+}}^{1} \approx \frac{1}{9} e^{i \frac{4 \pi}{3}} q^{-\frac{1}{3}}+\frac{5}{3}+6 e^{i \frac{2 \pi}{3}} q^{\frac{1}{3}}-\frac{76}{9} e^{i \frac{4 \pi}{3}} q^{\frac{2}{3}}+\ldots, \\
& Q_{d_{-}}^{1} \approx \frac{1}{9} e^{i \frac{2 \pi}{3}} q^{-\frac{1}{3}}+\frac{5}{3}+6 e^{i \frac{4 \pi}{3}} q^{\frac{1}{3}}-\frac{76}{9} e^{i \frac{2 \pi}{3}} q^{\frac{2}{3}}+\ldots
\end{aligned}
$$

and

$$
\begin{aligned}
& Q_{u}^{2} \approx 2+\ldots, \\
& Q_{t}^{2} \approx \frac{2}{9} q^{-\frac{1}{3}}-\frac{2}{3}+12 q^{\frac{1}{3}}-\frac{152}{9} q^{\frac{2}{3}}+\ldots, \\
& Q_{d_{+}}^{2} \approx \frac{2}{9} e^{i \frac{4 \pi}{3}} q^{-\frac{1}{3}}-\frac{2}{3}+12 e^{i \frac{2 \pi}{3}} q^{\frac{1}{3}}-\frac{152}{9} e^{i \frac{4 \pi}{3}} q^{\frac{2}{3}}+\ldots, \\
& Q_{d_{-}}^{2} \approx \frac{2}{9} e^{i \frac{2 \pi}{3}} q^{-\frac{1}{3}}-\frac{2}{3}+12 e^{i \frac{4 \pi}{3}} q^{\frac{1}{3}}-\frac{152}{9} e^{i \frac{2 \pi}{3}} q^{\frac{2}{3}}+\ldots
\end{aligned}
$$

The negative power $q^{-1 / 3}$ indicate that tachyonic states contribute to the charge functions $Q_{s}^{b}$ for the twisted sectors $s=t, d_{+}$and $d_{-}$. The positive powers result from massive string excitations. In fact a tower of massive string states contribute to these local shapes. This constitutes the most profound difference between the $\mathrm{SU}(9)$ and $\mathrm{E}_{7}$ models:

In the $\mathrm{E}_{7}$ model the tachyonic and massive string excitations give non-vanishing contributions, so the effective field theory calculation of the local profile of tadpole, which takes only the zero modes into account, is therefore questionable. The approximation that ignores the massive states can be justified because of the suppression factor of at least $\exp \left(-2 \pi \tau_{2} / 3\right)$. In contrast the tachyonic contribution are enhanced by the factor $\exp \left(2 \pi \tau_{2} / 3\right)$ so they could well give the leading effect. We will see below that they actually dominate the tadpole close to the orbifold fixed point, showing that effective field theory with zero modes ignores the most significant contributions near the orbifold singularity. For this comparison we do not need to compute the zero mode contributions again, since they are simply given by the profiles discussed in the previous subsection rescaled by the appropriate charges, which can be read from the constant terms in (16) and (17).

To investigate to what extent the tachyonic contributions to the tadpoles constitute the leading effect, we begin by defining the tachyonic momentum profile of the tadpole by

$$
T(k)=\int_{\mathcal{F}} \frac{\mathrm{d}^{2} \tau}{\tau_{2}^{2}} e^{-2 \pi i \frac{\tau_{1}}{3}} e^{2 \pi \frac{\tau_{2}}{3}}\left\{e^{-\Delta_{t}(\tau) \bar{k} k}+e^{i \frac{2 \pi}{3}} e^{-\Delta_{d_{-}}(\tau) \bar{k} k}+e^{i \frac{4 \pi}{3}} e^{-\Delta_{d_{+}}(\tau) \bar{k} k}\right\} .
$$

This is obtained by identifying the part of (8) that is proportional to $q^{-1 / 3}=\exp (-2 \pi i \tau / 3)$ for the $\mathrm{E}_{7}$ model. We must treat the tachyonic contributions of all twisted $\left(t, d_{+}\right.$and $\left.d_{-}\right)$sectors together a finite result: The integral over $\tau_{2}$ is divergent for each separately because the respective integrands are unbounded for large $\tau_{2}$. As the functions $\Delta_{s}(\tau)$ with $s=t, d_{ \pm}$all approach the normal ordering constant $c$ for large $\tau_{2}$ (see the approximations (10) or figures 2) the contributions cancel in this limit, by virtue of the $\mathbb{Z}_{3}$ projector identity

$$
\frac{1}{3}\left(1+e^{2 \pi i / 3}+e^{4 \pi i / 3}\right)=0 .
$$




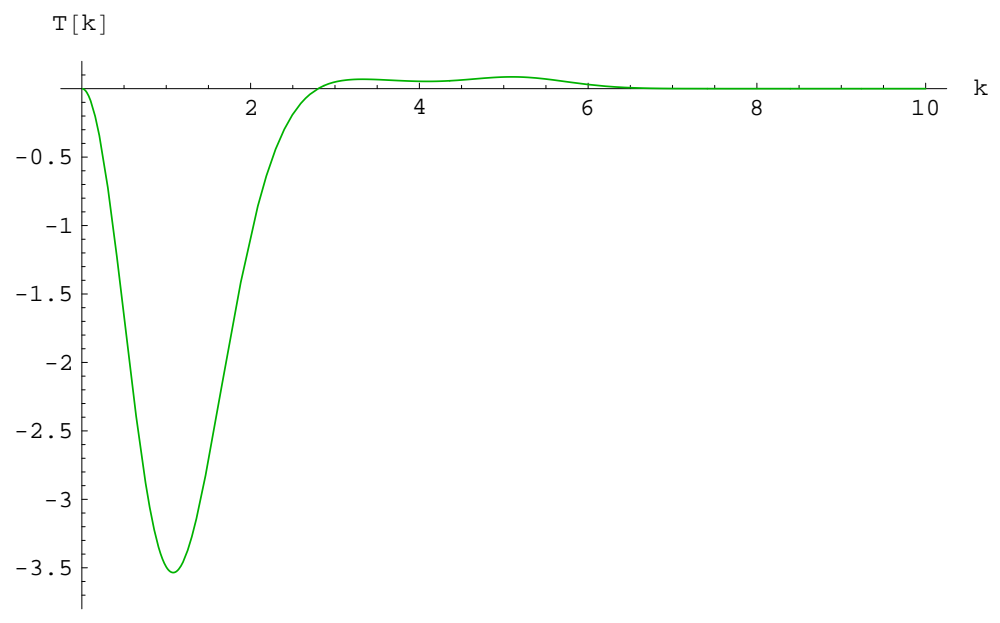

Figure 6: This plot shows the tachyonic contributions to the gauge field tadpole as a function of the absolute value of the internal momentum $k$.

This relation also implies that the tachyonic contributions vanish in the limit in which only the zero modes contribute, i.e. $k \rightarrow 0$. As the zero mode spectrum does not contain tachyons, the vanishing of $T(k)$ in this limit is the correct behavior. This suggests that it is more accurate to say that the tachyons cancel each other at the zero mode level, rather than that they are entirely absent. One might worry that because tachyonic states run around in the loop, one can describe the decay of a gauge field in two tachyons by cutting the diagram using the optical theorem. ${ }^{3}$ This is not the case because, as observed in section [3, the tadpole is real, hence its imaginary component associates this decay is zero.

This limiting behavior of the tachyonic contributions to the tadpole are readily verified in plot of figure 6] To understand the other major features of the shape of this tadpole, it is useful to rewrite equation (18) in a manifestly real form

$$
T(k)=\int_{\mathcal{F}} \frac{\mathrm{d}^{2} \tau}{\tau_{2}^{2}} e^{2 \pi \frac{\tau_{2}}{3}}\left\{\cos \left(\frac{2 \pi}{3} \tau_{1}\right)\left(e^{-\Delta_{t}(\tau) \bar{k} k}-E_{+}(k \mid \tau)\right)-\sqrt{3} \sin \left(\frac{2 \pi}{3} \tau_{1}\right) E_{-}(k \mid \tau)\right\} .
$$

We have introduced the even/odd functions

$$
E_{ \pm}(k \mid \tau)=\frac{1}{2}\left(e^{-\Delta_{d_{+}}(\tau) \bar{k} k} \pm e^{-\Delta_{d_{-}}(\tau) \bar{k} k}\right), \quad E_{ \pm}\left(k \mid-\tau_{1}, \tau_{2}\right)= \pm E_{ \pm}\left(k \mid \tau_{1}, \tau_{2}\right) .
$$

Using (12) it is straightforward to verify the parity under $\tau_{1} \rightarrow-\tau_{1}$, and that $E_{+}(0 \mid \tau)=1$ and $E_{-}(0 \mid \tau)=0$. Close to $k=0$ the term with $E_{-}$gives a negligible contributions compared to the first two terms. We know that $\Delta_{t}>\Delta_{d_{ \pm}}$and so $E_{+}(k \mid \tau)$ is bigger than the first term in (20) for non-zero $k$. Consequently, the integral first becomes more and more negative. However, for larger $k$ $E_{-}(k \mid \tau)$ becomes comparable to $E_{+}(k \mid \tau)$. As the integrals give the largest contributions where the propagators are the smallest, i.e. $\tau_{1} \approx \pm 1 / 2$, the $E_{-}(k \mid \tau)$ contributions dominates because of the factor $\sqrt{3} \sin \left(2 \pi \tau_{1} / 3\right) \approx \pm 3 / 2$. This means that the integrand turns positive, and for large values of $k$

\footnotetext{
${ }^{3}$ We are indebted to T. Gherghetta for raising this point.
} 


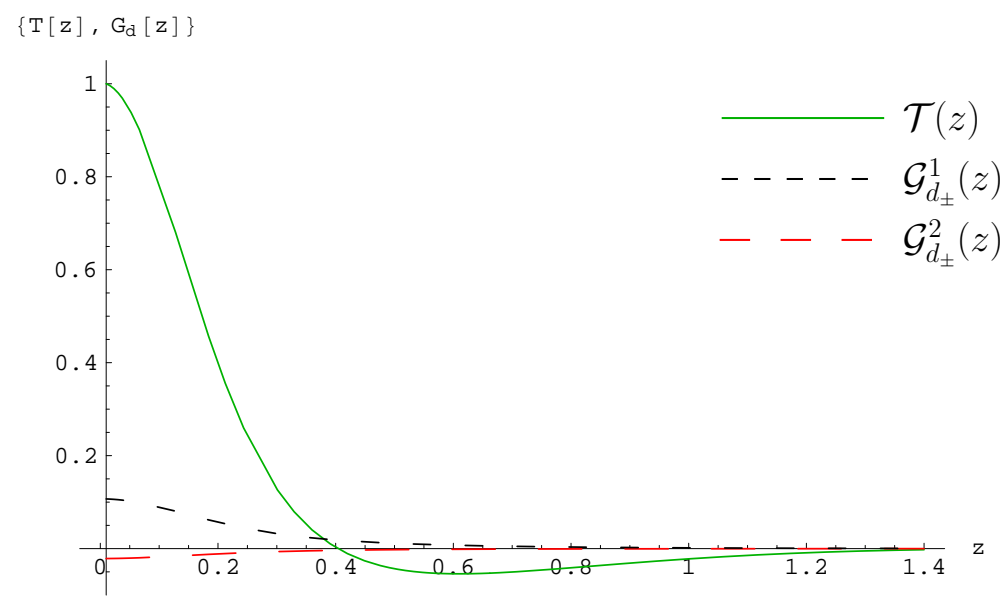

Figure 7: The coordinate space representation of the tachyonic contributions to the gauge field tadpole is displayed by the solid line. This contribution dominates the largest zero mode contribution coming from the $d_{ \pm}$sectors.

the integral takes positive values. As $k$ increases the exponential damping suppressed all contributions, and $T(k)$ tends to zero.

It is also instructive to study the tadpole due to the tachyons in the coordinate space representation. This tadpole can also be cast in a manifestly real form analogous to the expression (20):

$$
\mathcal{T}(z)=\int_{\mathcal{F}} \frac{\mathrm{d}^{2} \tau}{\tau_{2}^{2}} e^{2 \pi \frac{\tau_{2}}{3}}\left\{\cos \left(\frac{2 \pi}{3} \tau_{1}\right)\left(\frac{e^{-|z|^{2} / \Delta_{t}(\tau)}}{\left(\pi \Delta_{t}(\tau)\right)^{3}}-\mathcal{E}_{+}(k \mid \tau)\right)-\sqrt{3} \sin \left(\frac{2 \pi}{3} \tau_{1}\right) \mathcal{E}_{-}(k \mid \tau)\right\} .
$$

Like the exponential factor, the even and odd functions

$$
\mathcal{E}_{ \pm}(z \mid \tau)=\frac{1}{2}\left(\frac{e^{-|z|^{2} / \Delta_{d_{+}}(\tau)}}{\left(\pi \Delta_{d_{+}}(\tau)\right)^{3}} \pm \frac{e^{-|z|^{2} / \Delta_{d_{-}}(\tau)}}{\left(\pi \Delta_{d_{-}}(\tau)\right)^{3}}\right), \quad \mathcal{E}_{ \pm}\left(z \mid-\tau_{1}, \tau_{2}\right)= \pm \mathcal{E}_{ \pm}\left(z \mid \tau_{1}, \tau_{2}\right),
$$

contain the six dimensional Gaussian normalization factors $1 /\left(\pi \Delta_{d_{ \pm}}\right)^{3}$. Therefore, the integral over the orbifold of each of these exponentials is normalized to unity. It follows that the integrated tadpole due to tachyons vanish. This reflects the cancellation of the tachyonic contributions within the zero mode theory.

The full coordinate profile of the tachyonic contributions to the tadpole is depicted in figure $\mathbf{Z}$ by the solid green line. The main features can be explained by a similar analysis as performed above, but now taking the properties of the Gaussian normalization factor into account. For the behavior near $z=0$ this is already crucial, because contrary to the case above, $\mathcal{E}_{-}(z \mid \tau)$ does not vanish but is dominated by $1 /\left(\pi \Delta_{d_{ \pm}}(\tau)\right)^{3}$ with $\tau \approx(\mp 1+i \sqrt{3}) / 2$. Since there $\sqrt{3} \sin \left(2 \pi \tau_{1} / 3\right) \approx \mp 3 / 2$, the term with $\mathcal{E}_{-}(z \mid \tau)$ is the largest in (22) and positive. Since $1 / \Delta_{t}(\tau)$ is always smaller than $1 / \Delta_{d_{ \pm}}$the first exponential factor in the expression for the coordinate representation of the tadpole becomes the most important for large values of $|z|$. For even larger $|z|$ the exponential suppression forces the shape to approach zero. 
In figure 7 the tachyonic contributions to the tadpole is plotted together with the leading contributions from the zero modes. Inspecting figure 4 we infer that the twisted sectors $d_{ \pm}$give the largest contributions to the zero mode states. (The other sectors contributions are at least one order of magnitude less, and can therefore safely be ignored in the present analysis.) The normalization of the zero modes in the $d_{ \pm}$sectors compared to the tachyonic sector is 15 and -3 for the first and second $E_{8}$, respectively, see equations (16) and (17). These relative normalizations have been taken into account in comparison figure 7 . We conclude that the tachyonic states totally dominate the profile of the tadpoles for the U(1)'s in both Es's near the singularity.

\section{Discussion}

We devote the final section of this paper to a discussion of our string theory results for local gauge field tadpoles. We speculate as to how some of the stringy effects we encountered could be included in field theory.

In this work we have studied the structure of gauge field tadpoles in heterotic string on the (noncompact) orbifold $\mathbb{C}^{3} / \mathbb{Z}_{3}$, as recently computed in 1 . The tadpoles are given as an integral over the fundamental domain of a sum of charge functions times Gaussian distributions. The charge functions encode the states that contribute to the tadpole. By contrast the Gaussian distributions are universal in the sense that they affect the profiles of all participating states. The widths of these Gaussian distributions are determined by the expectation value of the square of the internal coordinate fields on the string world sheet. Since the shape of the fundamental domain dictates that $\tau_{2}>\sqrt{3} / 2$, the expansions in fractional powers of $\exp \left(-2 \pi \tau_{2}\right)$ are rapidly convergent.

By keeping only the zeroth order in this expansion of these Gaussian widths, we recovered the behavior of untwisted and twisted states in field theory up to the addition of some constants. For the twisted states these constants are particularly important, since they lead to a smooth cut-off for large internal momenta. This is in contrast to the naive field theory treatment of fixed point states which assumes that the contribution of any internal momentum is equally. When looking at the tadpole in a coordinate space representation, the effect of these constants is that the twisted states are not localized like delta functions at the fixed point, but rather are spread out around the orbifold singularity with widths comparable to a few string lengths. The string normal ordering constant has set a regularization scale for the orbifold singularity.

Because of the clear physical importance of these constants, we suggest that such constants should always be included in a field theoretical description of fixed point states. The transition from the string theory to the field theory treatment can be easily achieved by replacing the integration over the fundamental domain by an integration over Schwinger proper time. This methodology gives a well defined cut-off procedure because the string normal ordering constant acts as a regulator, that manifestly preserves Lorentz invariance.

A natural question therefore is whether these constants can be determined from first principals. In string theory these constants have the interpretation of normal ordering constants, which arise because the subtraction of singular terms on the string world sheet has an ambiguity. As we showed in [1] all tadpole widths depend on a single normal ordering constant. We have observed that this constant has to bounded from below. If this bound is violated, the target space description of the string theory breaks down because the Gaussian widths may become negative for some values of the modular parameters. We were not able to establish an upper bound on this constant, however, we 
have argued that the localization of the twisted states is the strongest if the lower bound is saturated. This most closely mimics the naive field theory description of the states at the fixed points.

These arguments show that we have some constraints on these constants that set the width of the twisted states over the internal dimensions from string theory. We doubt that direct field theoretical principles will allow us say much about them, because it is precisely the functional dependence on $\exp \left(-2 \pi \tau_{2}\right)$ of the widths that led to the lower bound in the string theory analysis. We find that the $\tau_{1}$ dependence of the widths $\Delta_{s}$ have the effect of increasing the lower bound for $c$.

As described above, the Gaussian distributions are only one aspect of the structure of the one-loop gauge field tadpoles. They also contain 'charges' which are functions of the modular parameter as well: Massless states give constant contributions, while massive string excitations give rise to some positive power of $\exp \left(-2 \pi \tau_{2}\right)$. However, unlike the widths of the Gaussian distributions, these are not the only corrections: In the model in which $\mathrm{E}_{8} \times \mathrm{E}_{8}{ }^{\prime}$ is broken to $\mathrm{E}_{7} \times \mathrm{U}(1) \times \mathrm{SO}(14)^{\prime} \times \mathrm{U}(1)^{\prime}$, we found that there are also tachyons contributing with a growing exponential $\exp \left(2 \pi \tau_{2} / 3\right)$. This does not constitute a contradiction with the statement that heterotic string theories on orbifold do not contain tachyons in their spectrum: The tachyons contributing to the tadpole are not physical particles as they only exist as off-shell quantum corrections in the loop. Moreover, we showed that when the tadpole is integrated over the orbifold, the tachyonic contributions cancel each other. As figure 7 showed they only give contributions in the vicinity of the orbifold singularity, but there they completely dominate all other massless and massive string contributions!

This remarkable result that tachyons in some string models can give sizable contributions to local operators, like gauge field tadpoles, is somewhat provocative. Normally, by considering the zero modes of the string, one assumes that one captures the major part of the perturbative quantum corrections. Here we see that for local physics the tachyons sometimes play the most important role. In string theory these tachyonic contributions can be identified as straightforwardly as the massless or massive string excitations. On the other hand, in field theory it is not all obvious when these local tachyonic contributions should be included, as it is not simply the presence of an anomalous $U(1)$ which requires that they should be considered. Therefore, further research on local tachyonic corrections is necessary to gain a more complete understanding of their relevance.

\section{Acknowledgements}

We would like to thank T. Gherghetta for useful discussions. The work of S.G.N. was partially supported by DOE grant DE-FG02-94ER-40823. The work of M.L. was partially supported by NSERC Canada. S.G.N. would like to thank the University of British Columbia for their hospitality during which this project got started. M.L. wishes to thank the University of Minnesota for their hospitality during part of the preparation of this work.

\section{References}

[1] S. Groot Nibbelink and M. Laidlaw "Stringy shape of singularities: I. heterotic gauge field tadpoles". hep-th/0311013.

[2] L. Randall and R. Sundrum "A large mass hierarchy from a small extra dimension" Phys. Rev. Lett. 83 (1999) 3370-3373 hep-ph/9905221 
[3] L. Randall and R. Sundrum "An alternative to compactification" Phys. Rev. Lett. 83 (1999) 4690-4693 hep-th/9906064

[4] N. Arkani-Hamed, S. Dimopoulos, and G. R. Dvali "The hierarchy problem and new dimensions at a millimeter" Phys. Lett. B429 (1998) 263-272 hep-ph/9803315

[5] R. Barbieri, L. J. Hall, and Y. Nomura "A constrained standard model from a compact extra dimension" Phys. Rev. D63 (2001) 105007 hep-ph/0011311.

[6] J. Polchinski "Evaluation of the one loop string path integral" Commun. Math. Phys. 104 (1986) 37.

[7] D. M. Ghilencea, H. P. Nilles, and S. Stieberger "Divergences in Kaluza-Klein models and their string regularization" New J. Phys. 4 (2002) 15 hep-th/0108183

[8] P. Fayet and J. Iliopoulos "Spontaneously broken supergauge symmetries and Goldstone spinors" Phys. Lett. B51 (1974) 461-464.

[9] E. A. Mirabelli and M. E. Peskin "Transmission of supersymmetry breaking from a 4-dimensional boundary" Phys. Rev. D58 (1998) 065002 hep-th/9712214

[10] D. M. Ghilencea, S. Groot Nibbelink, and H. P. Nilles "Gauge corrections and FI-term in 5D KK theories" Nucl. Phys. B619 (2001) 385-395 hep-th/0108184

[11] R. Barbieri, L. J. Hall, and Y. Nomura "A constrained standard model: Effects of Fayet-Iliopoulos terms" hep-ph/0110102

[12] C. A. Scrucca, M. Serone, L. Silvestrini, and F. Zwirner "Anomalies in orbifold field theories" Phys. Lett. B525 (2002) 169-174 hep-th/0110073

[13] S. Groot Nibbelink, H. P. Nilles, and M. Olechowski "Spontaneous localization of bulk matter fields" Phys. Lett. B536 (2002) 270-276 hep-th/0203055

[14] S. Groot Nibbelink, H. P. Nilles, and M. Olechowski "Instabilities of bulk fields and anomalies on orbifolds" Nucl. Phys. B640 (2002) 171-201 hep-th/0205012

[15] H. M. Lee, H. P. Nilles, and M. Zucker "Spontaneous localization of bulk fields: The six- dimensional case" hep-th/0309195

[16] R. Barbieri, R. Contino, P. Creminelli, R. Rattazzi, and C. A. Scrucca "Anomalies, Fayet-Iliopoulos terms and the consistency of orbifold field theories" Phys. Rev. D66 (2002) 024025 hep-th/0203039

[17] D. Marti and A. Pomarol "Fayet-Iliopoulos terms in 5d theories and their phenomenological implications" Phys. Rev. D66 (2002) 125005 hep-ph/0205034

[18] G. von Gersdorff, N. Irges, and M. Quiros "Radiative brane-mass terms in $D>5$ orbifold gauge theories" Phys. Lett. B551 (2003) 351-359 hep-ph/0210134

[19] C. Csaki, C. Grojean, and H. Murayama "Standard model Higgs from higher dimensional gauge fields" Phys. Rev. D67 (2003) 085012 hep-ph/0210133.

[20] S. Groot Nibbelink, H. P. Nilles, M. Olechowski, and M. G. A. Walter "Localized tadpoles of anomalous heterotic U(1)'s" Nucl. Phys. B665 (2003) 236-272 hep-th/0303101

[21] M. Dine, N. Seiberg, and E. Witten "Fayet-Iliopoulos terms in string theory" Nucl. Phys. B289 (1987) 589.

[22] J. J. Atick, L. J. Dixon, and A. Sen "String calculation of Fayet-Iliopoulos D terms in arbitrary supersymmetric compactifications" Nucl. Phys. B292 (1987) 109-149.

[23] M. Dine, I. Ichinose, and N. Seiberg "F terms and D terms in string theory" Nucl. Phys. B293 (1987) 253. 
[24] E. Poppitz "On the one loop Fayet-Iliopoulos term in chiral four dimensional type I orbifolds" Nucl. Phys. B542 (1999) 31-44 hep-th/9810010

[25] F. Gmeiner, S. Groot Nibbelink, H. P. Nilles, M. Olechowski, and M. G. A. Walter "Localized anomalies in heterotic orbifolds" Nucl. Phys. B648 (2003) 35-68 hep-th/0208146

[26] M. B. Green and J. H. Schwarz "Anomaly cancellation in supersymmetric d=10 gauge theory and superstring theory" Phys. Lett. B149 (1984) 117-122.

[27] S. Groot Nibbelink "Traces on orbifolds: Anomalies and one-loop amplitudes" JHEP 07 (2003) 011 hep-th/0305139 